\section{Breve esbozo de una propuesta para la inclusión de socios trabajadores de las Cooperativas Especializadas de Trabajo en el Sistema de Seguridad Social del Paraguay}

Ana Leticia Carosini Ruiz-Díaz

Docente tutora de la Facultad

de Ciencias Económicas, Universidad

Nacional de Asunción, Paraguay.
Economía Social y Solidaria /

Perspectivas

RECEPCIÓN: 26/06/15

ACEPTACIÓN FINAL: 13/08/15

\section{Resumen}

El artículo se deriva de la tesis presentada en la Maestría Oficial en Economía Social, Cooperativas y Entidades No Lucrativas de la Universidad de Valencia, España, en la cual se aborda una problematización sobre políticas públicas vinculadas a la seguridad social en el Paraguay y la inclusión de los socios de las Cooperativas Especializadas de Trabajo (CET). La pregunta de investigación es la siguiente: ¿es posible estructurar el sistema de seguridad social en Paraguay de manera que incluya a los socios trabajadores de las CET?, y para responderla se traza como objetivo proponer lineamientos básicos para un Seguro Social que incluyan a los socios trabajadores de las CET en el Paraguay sobre la base del modelo español. Con un nivel de investigación exploratorio, descriptivo, explicativo y propositivo, se ha logrado analizar la situación de las CET desde el punto de vista legal e institucional en el país y esbozar algunos lineamientos principales para una intercooperación de la Economía Social y Solidaria (ESS).

Palabras clave

- cooperativas

- trabajo

- inclusión

- seguridad social

\section{Resumo}

O artigo é derivado da tese apresentada no Master Oficial da Economia Social, Cooperativas e Organizações sem fins lucrativos da Universidade de Valencia, Espanha, que lida com uma problemática sobre políticas públicas relacionadas com a segurança social no Paraguai e a inclusão de parceiros das Cooperativas Especializadas de Trabalho (CET). A pergunta da pesquisa é: É possível estruturar o sistema de segurança social no Paraguai de forma de incluir parceiros trabalhadores das CET ? Para responder é considerado alvo propor diretrizes básicas para a Segurança Social que incluam aos parceiros trabalhadores das CET no Paraguai, com base no modelo espanhol. Com um nível de pesquisa exploratório, descritivo, explicativo e proposital se conseguiu analisar a situação das CET do ponto de vista jurídico e institucional no país e delinear algumas diretrizes principais para uma intercooperação da Economia Social e Solidária.(ESS).

Palavras-chave

- cooperativas

- trabalho

- inclusão

- segurança social
Para citación de este artículo

Carosini Ruiz-Díaz, A.L. (2015). Breve esbozo de una propuesta para la inclusión de socios trabajadores de las Cooperativas Especializadas de Trabajo en el Sistema de Seguridad Social del Paraguay. En Revista $+E$ versión digital, (5), pp. 76-83. Santa Fe, Argentina: Ediciones UNL. 


\section{Introducción}

La seguridad social, entendida como las medidas de protección que brinda la sociedad a sus miembros en caso de diversas circunstancias que los afectan, es considerada desde el siglo XIX y ha evolucionado hasta la actualidad. La importancia para la Organización Internacional del Trabajo (OIT) se comprueba en el año 1952 al firmarse el Convenio 102, que es reputado como la norma mínima en lo relativo a la seguridad social. Es el

"único convenio internacional que define las nueve ramas de la seguridad social, establece normas mínimas para cada una de ellas, y enuncia principios para la sostenibilidad y buena gobernanza de dichos sistemas" (OIT, 2011:14).

A fin de actualizar la discusión sobre el tema, y teniendo en cuenta la necesidad de actualización del Convenio 102/1952, en la Conferencia Internacional del Trabajo en junio de 2001 fue adoptada una resolución en la cual no sólo se confirma el papel de la seguridad social como un derecho humano sino también como una necesidad social y económica para todos los países, independientemente de su nivel de desarrollo. El Paraguay es uno de los pocos países de América del Sur, y el único del Mercado Común del Sur (Mercosur), que no ha ratificado dicho Convenio de la OIT. Sin embargo, en la Constitución Nacional del año 1992 se establece el Sistema de Seguridad Social en el Paraguay. Y la legislación complementaria a dicha norma ha definido y creado los organismos encargados de la prestación de los servicios del sistema regulado de jubilaciones y pensiones.

El sistema de pensiones y jubilaciones paraguayo está integrado por ocho regímenes o entes regulados y seis entidades privadas no reguladas, destacándose las instituciones del sector público, denominadas Instituto de Previsión Social (IPS) y Caja Fiscal, como las que aglutinan a la mayoría de los cotizantes, entre los que se encuentran incluidos todos los trabajadores en relación directa del sector privado y entes descentralizados, docentes de los sectores público y privado, trabajadores domésticos, jubilados y pensionados y docentes jubilados. El IPS es la principal institución del Sistema de Seguridad Social en Paraguay, cuyo sistema de

reparto cuenta con recursos que se conforman del aporte tripartito: empleado, empleador y Estado.

Aun con esta estructura, los datos demográficos extraídos de la Encuesta Permanente de Hogares (EPH) 2011 de la Dirección General de Estadísticas, Encuestas y Censos (DGEEC) evidencian que aproximadamente el 39,3\% de las personas asalariadas o dependientes está acogido a un sistema de jubilación o pensión, en tanto son las mujeres quienes presentan mayor cobertura de jubilación respecto de los hombres (50,9 \% y 33,9 \%, respectivamente) (DGEEC, 2011:1). Estos niveles de cobertura, si bien se encuentran dentro del rango estimado por estudios de la OIT para los países de la región latinoamericana, ${ }^{1}$ también manifiestan la problemática de incluir en dicho porcentaje sólo a trabajadores del sector formal, sin brindar posibilidades de inclusión efectiva a la mano de obra del vasto sector informal que existe en el país. Esta situación es más preocupante en el sector cooperativo del Paraguay, considerando que el cooperativismo paraguayo presenta una situación similar a la explicada en párrafos anteriores en el ámbito de la seguridad social. Esto se evidencia en el hecho de que sólo los trabajadores no socios de las cooperativas son los que pueden tener acceso al sistema de seguro social por ser considerados trabajadores dependientes; mientras que los socios trabajadores no pueden acceder debido a la interpretación legal de que los mismos son considerados como dueños, socios o propietarios de las cooperativas; es decir, no se encuentran en situación de dependencia laboral. Por ello, se evidencia la necesidad de un marco inclusivo de la seguridad social, más aún en aquellas cooperativas que se dedican exclusivamente a ofrecer mano de obra, denominadas Cooperativas Especializadas de Trabajo (CET).

1) En América Latina y el Caribe, cuya tradición de seguridad social está muy arraigada, en la mayoría de los casos la cobertura refleja la proporción de las personas que trabajan en la econo- mía formal (del 30 al $60 \%$, excepto en algunas islas del Caribe donde el grado de formalización de la economía es mayor) (OIT, 2013:57). 





Es por ello que en el presente trabajo de investigación se plantean preguntas guía como las siguientes: ¿es posible estructurar el sistema de seguridad social en Paraguay de manera que incluya a los socios trabajadores de las CET? ¿Cuáles son los factores del sistema de seguridad social en países como España que podrían ser considerados para lograr un sistema de seguridad social inclusivo, con respecto a los socios trabajadores de las cooperativas de trabajo, en Paraguay? A fin de lograr responder a estas preguntas, se ha trazado como objetivo general de la investigación el de proponer lineamientos básicos a ser considerados para estructurar un Seguro Social que incluya a los socios trabajadores de las CET en el Paraguay sobre la base del modelo de seguridad social desarrollado en España. La importancia de este trabajo reside en que pretende brindar un estudio pionero sobre la necesidad de estructurar un Sistema de Seguridad Social inclusivo que incorpore a aquella parte de la población paraguaya que se encuentra asociada a CET.

\section{Metodología del trabajo realizado}

La investigación adopta un enfoque exploratorio, descriptivo, explicativo y propositivo. Se destaca que este trabajo es exploratorio, considerando que en el Paraguay son incipientes los estudios sobre el sector cooperativo nacional, y sobre las Cooperativas de Trabajo en particular. En este sentido, este estudio podría considerarse como pionero en el análisis de la relación entre sector cooperativo y la seguridad social en el país.

El enfoque adoptado es descriptivo dado que se presentan los Sistemas de Seguridad Social en España y Paraguay con miras a identificar lineamientos básicos para estructurar un seguro social inclusivo. Con el enfoque explicativo propositivo se pretende analizar la caracterización de los sistemas nacionales señalados, a fin de estructurar una propuesta de lineamientos a considerar en la construcción de un modelo inclusivo de seguridad social para el caso paraguayo. Los métodos utilizados son: deductivo, analítico y sintético, se aplicaron las técnicas de revisión bibliográfica documental y se recurrió a documentación relevante a nivel nacional e internacional. En el ámbito nacional, se consideraron informaciones del sector cooperativo a nivel de la Confederación Paraguaya de Cooperativas (CONPACOOP) y del Instituto Nacional de Cooperativismo (INCOOP) En el ámbito internacional, de la Organización Internacional del Trabajo (OIT), del Centro Internacional de Investigación e Información sobre la Economía Pública, Social y Cooperativa (CIRIEC), entre otros. Para comprender el Sistema de Seguridad Social vigente en España se ha realizado la revisión bibliográfica de libros, revistas especializadas, así como de las documentaciones oficiales de la Unión Europea, del Ministerio de Empleo y Seguridad Social de España, entre otros.

\section{Resultados y discusiones sobre el análisis de la inclusión de los socios de las CET en el Sistema de Seguridad Social en Paraguay}

El Paraguay se caracteriza por tener una población económicamente activa joven, lo cual impacta aún más en la definición de políticas oportunas y adecuadas al bono demográfico que implica el porcentaje significativo de mano de obra joven. Además cuenta con un sector cooperativo de gran importancia, considerando que desde la crisis financiera nacional del año 1995 ha logrado un crecimiento significativo, lo cual ha evidenciado más la necesidad de un marco normativo adecuado, dentro del cual se encuentra el relativo a la seguridad social, principalmente en aquellas cooperativas que se dedican exclusivamente a ofrecer servicios laborales.

Las cooperativas de trabajo en el Paraguay han experimentado una mayor difusión a partir de los estudios realizados por la CONPACOOP desde el año 2007, entre los que se halla el de Sonia Morínigo (2011), con un relevamiento sobre la Cobertura de Seguro Médico, de Vida y Jubilatorio para los socios de las cooperativas de trabajo. Para entender la conceptualización de las cooperativas de trabajo en el Paraguay se recurre a las normas del sector, en las cuales se establece que las Cooperativas de Trabajo se integran con el objeto de dar empleo a sus socios (Decreto № 14052/1996, artículo 109), que en dichas cooperativas los socios no tienen relación de dependencia laboral (Ley $N^{\circ}$ 438/1994, artículo 8); y que, sin perjuicio de la diversidad de tareas, los socios tendrán obligación indelegable e intransferible de trabajar personalmente en la prestación que le fuere asignada (Resolución INCOOP N ${ }^{\circ}$ 7110/2011, artículo 3.e).

En cuanto a la regulación de las acciones en las CET, se destaca que la regulación externa de los actos cooperativos está regida por la Ley de Cooperativas y subsidiariamente al derecho común. (Ley N 438/1994, artículo 8); mientras que en la regulación interna se establece un Reglamento General de Trabajo a ser elaborado por la entidad y aprobado por la Asamblea de socios, en virtud del cual se suscribirá un contrato individual en el que se estipularán las condiciones particulares relacionadas con la prestación de servicios (Resolución INCOOP № 7110/2011, artículo 3.a, 3.b).

En ese sentido, tanto las características del país como las mismas intenciones de cooperación han sido pilares fundamentales que promovieron la organización de nuevos tipos de cooperativas, entre las que se encuentran las especializadas en trabajo, las cuales podrían ser una alternativa efectiva que permita la disminución de las altas tasas de desempleo, inestabilidad, dependencia laboral o quizá de las mismas tasas de migración que se han observado a lo largo de los últimos años. Dichas cooperativas cuentan con socios que se desempeñan como trabajadores independientes, autónomos o cuentapropistas, razón por la que no se encuentran incluidos en el Sistema de Seguridad Social paraguayo. Conforme lo expuesto por Caballero (2011), el Sistema de 
Seguridad Social en Paraguay presenta una heterogeneidad entre las diversas cajas previsionales, así como la necesidad de inclusión de sectores de la población que no están incluidos. El Instituto de Previsión Social (IPS) es la entidad más importante del sistema de seguridad, la cobertura se extiende a todos los asalariados del sector formal del país, personal de entes descentralizados del Estado o empresas mixtas, maestros de los sectores público y privado y el servicio doméstico. Los beneficios son extensibles a los cónyuges o convivientes, hijos y padres del asegurado en situación de dependencia. Ha experimentado reformas en cuanto a ampliación de la cobertura, llegando incluso a la propuesta para incluir a trabajadores independientes.

Autores como Rodríguez y Pei (2003), así como Holst (2003), han indicado que el sector privado paraguayo en materia de servicios jubilatorios y médicos se basa en un sistema que no se encuentra regulado, aunque está más modernizado que el sector público al adoptar el sistema de capitalización en los servicios jubilatorios brindados principalmente por las cajas mutuales. El sistema es flexible y programado, permite al asociado acogerse a la jubilación que más le convenga y puede incrementar su aporte ordinario o realizar aportes extraordinarios en cualquier momento.

Al realizar el análisis comparativo con el Sistema de Seguridad Social existente en España, basado en autores como Sánchez Martínez (2007) y Ruesga Benito (2009), se evidencia que dicho sistema fue construido sobre la base de dos ámbitos o niveles de protección principales. Existe un primer nivel de protección asistencial o de naturaleza no contributiva a cuya acción protectora acceden todos los ciudadanos por la mera condición de serlo, cuando adicionalmente concurre en ellos una situación de necesidad (falta de ingresos) y que se financia con aportaciones del Estado de origen tributario general. Junto a éste, un segundo nivel de protección denominado contributivo, mucho más importante en términos cuantitativos, que tiene como objeto la protección de carácter profesional y que se financia precisamente mediante las cotizaciones sociales derivadas de la actividad laboral de los trabajadores afiliados y de los empresarios. El análisis realizado sobre el caso español fundado en la legislación vigente en España, y autores como López Gandía (2006), con respecto al Sistema de Seguridad Social, muestra que los socios trabajadores de las Cooperativas de Trabajo Asociado (CTA) tienen la posibilidad de integrarse, dependiendo de la actividad realizada, como asimilados a trabajadores por cuenta ajena o como trabajadores por cuenta propia, opción que se reflejará en los estatutos de la cooperativa. Para realizar una buena elección se precisa analizar aspectos como: requisitos de entrada, tipología de las prestaciones, años de cotización, cuantía de las prestaciones, entre otros (Betzuen et al., 1993:19). En este contexto, en el Paraguay es importante analizar los factores o lineamientos a considerar a fin de lograr la inclusión efectiva de los socios trabajadores de las CET en el Sistema de Seguridad Social. Dichas consideraciones son las siguientes: a) Propuestas para el marco normativo paraguayo: los lineamientos a considerar en la normativa paraguaya se inician desde la definición misma de una cooperativa de trabajo. En cuanto a la vinculación entre los sistemas de seguridad español y paraguayo, en primer lugar, se aclara que en ambos países, en el ámbito de las Cooperativas de Trabajo Asociado en España y CET en Paraguay, la población objetivo del sistema se refiere al socio trabajador miembro de la cooperativa. En segundo lugar, la norma cooperativa es la que rige el relacionamiento laboral a través de un reglamento de trabajo o contrato societario. Sin embargo, en Paraguay existe una ambigüedad dada por el hecho de que se especifica que las relaciones entre las cooperativas, en general, y sus empleados y obreros, se rigen por la legislación laboral, aun cuando también se señala, en el caso de las cooperativas de trabajo, que los socios no tienen relación de dependencia laboral. Asimismo, estipula que la contratación excepcional de terceros no socios se regirá por el Código Laboral o Código Civil (Resolución INCOOP № 7110/2011, artículos 2 y 3.g). En este sentido, como lo expresa García Jiménez (2008:263), un reglamento de trabajo debe contener no sólo lo establecido en los estatutos o acuerdos asamblearios sino también respetar las disposiciones reguladoras de este tipo de cooperativas así como los derechos y garantías legalmente establecidos en el derecho laboral común.

Por lo anterior, es importante establecer una normativa coherente en el sector cooperativo paraguayo, que determine específicamente la relación societaria en dicho sector, evitando ambigüedades o confusión en el relacionamiento de trabajo cooperativo. Asimismo, dada la particularidad de las cooperativas de trabajo en el Paraguay, la disposición adoptada en la legislación española con respecto a que los socios trabajadores de dicho tipo de cooperativas pueden adoptar la modalidad de trabajadores por cuenta propia o ajena, según sea el caso de su actividad laboral, constituye una disposición racional. Fundamentalmente, porque en el caso paraguayo las cooperativas de trabajo, por definición legal, se constituyen para brindar empleo a sus socios, que en la mayoría de los casos responden a características de trabajadores independientes o autónomos. En Paraguay se visualiza la inexistencia un marco normativo específico con respecto al tema de la protección social, por lo cual se precisa que en el país se estudie y estructure un marco normativo actualizado a las necesidades del sector cooperativo, que abarque la problemática de la seguridad social. Más aún cuando la reglamentación vigente para las CET, que es la Resolución INCOOP № 7110/2011, especifica sobre componentes de la protección social que el seguro médico será obligatorio e indica como posibilidad, sujeta a decisión asamblearia, la contratación del seguro de vida y jubilatorio. Por ello, se sugiere modificar la Resolución INCOOP N 7110/2011, o bien derogar dicha resolución, y se propone la elaboración de una ley específica para las cooperativas de trabajo, como se ha hecho en los casos de las Leyes de Mutuales y de Cooperativas de Vivienda. 
La normativa general de la seguridad social en el país, tanto a nivel nacional como cooperativo, requiere de revisiones y modificaciones, atento a la realidad del mercado laboral paraguayo y teniendo en cuenta que la protección social es competencia del Estado. Así, se podría considerar modificar la Ley de Cooperativas, № 438/1994, juntamente con el Decreto Reglamentario, adicionando normas específicas para cada sector en cuanto al desarrollo de su competencia, sumado al hecho de que el país precisa de una reforma de todo el Sistema de Seguridad Social, la cual, aunque es prioritaria, se encuentra en proceso de estudio.

b) Propuesta para el marco institucional paraguayo: la Ley $N^{\circ} 2157 / 2003$, artículo $5^{\circ}$, establece que el INCOOP cuenta con órganos de fiscalización pública en el ámbito de su competencia, los cuales deberán coordinar y canalizar a través de la Dirección de Supervisión y Fiscalización del INCOOP, el cumplimiento de la Ley de Cooperativas, la citada ley y las demás vigentes en la materia. En este contexto, CONPACOOP, como entidad de integración de tercer grado del sector cooperativo, ha avanzado en realizar estudios exploratorios sobre diversos aspectos de la seguridad social, y conforme a los resultados de dichos estudios se evidencia la necesidad de potenciar la intercooperación entre entidades cooperativas del Paraguay, principalmente en el área de la seguridad social. Esto teniendo en cuenta que en el país existen entidades de la economía social que brindan servicios jubilatorios (mutuales) y médicos (cooperativas), en forma individual y sectorizada. De la experiencia española se desprende, para el caso paraguayo, que la institucionalidad de un Sistema de Seguridad Social precisa contar con entidades gubernamentales orientadas al tema. En este sentido, en Paraguay, a partir del año 2013, se cuenta con el denominado Ministerio de Trabajo, Empleo y Seguridad Social, entidad en la cual está inserta una Dirección de Seguridad Social que hasta la fecha se halla en proceso de fortalecimiento institucional. Asimismo, en el sector cooperativo, ante iniciativas de CONPACOOP de llevar a cabo proyectos y estudios, se requiere que la entidad asuma el liderazgo de socializar e implementar las recomendaciones resultantes de los mismos, tendientes a construir las bases para un cambio de mentalidad cooperativa hacia la intercooperación. Intercooperación para establecer complementariedad con EES, como alternativa para construir un modelo inclusivo en el Sistema de Seguridad Social en Paraguay. Ante lo expuesto sobre el sector cooperativo y su situación en cuanto a tenencia de seguro médico, de vida y jubilatorio, desde esta investigación se plantea una interrogante sobre la visualización de la alternativa de crear un Seguro Social Cooperativo. Esta posibilidad de considerar que el sector cooperativo cuenta con entidades que proveen servicios médicos, realiza operaciones de seguro de vida y también presta servicios jubilatorios, como es el caso de la Caja Mutual de Cooperativistas. Es así que llegar a estructurar un Seguro Social Cooperativo, como alternativa al Sistema de Seguridad Social vigente en el país, necesita una concienciación y entendimiento del sexto principio cooperativo, el de la intercooperación, en el entendimiento de que dicho principio se refiere a la necesidad que tienen las entidades cooperativas de trabajar conjuntamente mediante estructuras locales, nacionales, regionales e internacionales en un tema específico, y en el caso paraguayo sería el de la seguridad social. Como hasta la fecha no se han presentado casos concretos y exitosos de actividades conjuntas entre cooperativas paraguayas para lograr la profundización señalada en el accionar intercooperativo en el ámbito de servicios de seguro social, los expertos opinaron que se requiere un cambio de mentalidad basado en la vivencia de los principios cooperativos, desde las mismas autoridades hasta los niveles técnicos y de asociados del sector cooperativo paraguayo.

La opción de establecer un sistema complementario al régimen oficial de protección social implica que las mutualidades, no sólo la Caja Mutual de Cooperativistas, sino las demás que están relacionadas con sectores determinados, cuenten con el marco legal que posibilite la actuación complementaria entre dichas entidades. Asimismo, los estudios exploratorios existentes en CONPACOOP sobre el tema merecen ser profundizados con productos específicos de proyectos en cuanto a la visualización de los costos y beneficios de la intercooperación entre las diversas EES que brindan servicios médicos, logrando implementar efectivamente lo establecido como obligatoriedad en la Resolución del INCOOP para el caso de las CET. En este sentido, las limitaciones con respecto a la construcción de una base de datos que contenga informaciones sobre el sector cooperativo nacional, precisa de que el ente regulador, INCOOP, quien detenta la responsabilidad en dicho tema, pueda implementar efectivamente no sólo la elaboración y presentación del Balance Social, sino también de los diversos sistemas que actualmente se encuentran en la fase de elaboración y planificación de los mismos. El tema de la complementariedad en el caso paraguayo también precisaría de una nueva normativa que reglamente dicho sistema. La citada norma tendría que ser elaborada en el marco de la cooperación y trabajo interinstitucional entre las entidades públicas: MTEySS, IPS, y las del sector cooperativo: INCOOP, CONPACOOP, cajas mutuales, entre otras entidades de la economía social en Paraguay.

\section{Conclusiones y recomendaciones}

En el caso paraguayo existen propuestas de leyes que tienden a subsanar la situación de exclusión, específicamente en lo referente a servicios jubilatorios, aunque el análisis de las mismas determine que precisan seguir algunos lineamientos enmarcados en el avance que presenta la legislación española al respecto. Dichos lineamientos se centran en varios aspectos: la armonización de conceptos y unificación de términos, no sólo en cuanto a lo que se entienda por cooperativas de trabajo sino también el término abarcador de trabajador autónomo; el marco legal e institucional necesario para 


\section{6}

llegar a estructurar un Seguro Social

Cooperativo, como alternativa al

Sistema de Seguridad Social vigente

en el país, necesita una concienciación

y entendimiento del sexto principio

cooperativo, el de la intercooperación implementar eficientemente la inclusión de los socios trabajadores, centrado no solamente en lograr coherencia en la estructura legal sino en contar con los mecanismos institucionales que posibiliten dicha eficiencia. Unido a lo anterior, se plantean en esa investigación dos alternativas. Una, reside en utilizar el Sistema de Seguridad Social vigente, ampliando la cobertura del IPS, principal institución previsional, para lo cual es perentoria la realización de reformas en dicha entidad; y la otra alternativa consiste en lograr la intercooperación de las EES del cooperativismo paraguayo a fin de potenciar la implementación de un seguro social del sector, ya que cuenta con entidades de la economía social que brindan servicios jubilatorios, como de salud. Ambas propuestas, a fin de ser implementadas, requieren contar con los recursos para ello, lo cual implica superar algunas limitaciones y tomar medidas por parte de las autoridades de cada sector. Es decir, de parte del sector de la economía social, CONPACOOP precisa asumir el liderazgo para fomentar la intercooperación; y en el sector público, el IPS debe llevar a cabo las recomendaciones surgidas de estudios a nivel internacional con el objetivo de contar con una buena base de datos así como la infraestructura para hacer frente a una posible ampliación de cotizantes y beneficiarios en el mediano plazo.
Esta investigación, realizada en forma exploratoria, presenta una base interesante para posibles temas de investigación a futuro, como la viabilidad financiera del sistema de reparto de IPS, el análisis de costo-beneficios de la estructuración de un Seguro Social Cooperativo en el Paraguay, entre otros temas. Es por ello que, en el marco del proceso de integración regional, los desafíos de la economía globalizada traen como consecuencia generalizada, para economías pequeñas y abiertas como el Paraguay, el impacto de los sucesos externos que vuelve urgente la toma de decisiones en política. En este contexto, la economía paraguaya requiere propuestas que permitan superar determinados fenómenos que se han profundizado en los últimos años, como son la migración y la escasa generación de empleo productivo. Es así que se manifiesta la importancia del sector cooperativo como una forma de paliar la desocupación y la escasa inserción laboral de algunos sectores sociales y económicos, y se visibiliza a las cooperativas especializadas de trabajo como una alternativa válida no sólo para facilitar la ocupación laboral sino también para aumentar la generación de empleo y remuneraciones dignas. Es por eso que además se deben generar los instrumentos y sistemas adecuados para tornar dicha posibilidad en una realidad en el Paraguay. 


\section{Referencias bibliográficas}

Betzuen, A.; Bilbao, A. y De la Peña, I. (1993). La problemática de la seguridad social en el marco de las cooperativas de trabajo asociado. Bilbao: Instituto de Estudios Financieros-actuariales.

Caballero, M. (2011). Sistema Jubilatorio en el Paraguay. Sostenibilidad Financiera. Exposición realizada en el Primer Seminario Nacional de Seguridad Social. Caja de Jubilaciones y Pensiones del Personal de la Itaipú Binacional (CAJUBI). 8 y 9 de noviembre de 2011. Ciudad del Este, Paraguay.

Dirección General de Estadísticas, Encuestas y Censos (DGEEC) (2011). Principales indicadores de empleo e ingresos. EPH/2011. Encuestas Permanente de Hogares. Asunción, Paraguay.

García Jiménez, M. (2008). Autoempleo: trabajo asociado y trabajo autónomo. Madrid: Tecnos

Holst, J. (2003). La protección social en Paraguay. Condiciones, problemas, desafíos y perspectivas de los sistemas de previsión. Proyecto Planificación del Desarrollo Sostenible (STP-PLANDES). Asunción: Secretaría Técnica de Planificación. Instituto Nacional de Cooperativismo (INCOOP) (2008). Marco Legal y Regulatorio de las cooperativas en Paraguay. Asunción: INCOOP.

López Gandía, J. (2006). Las cooperativas de trabajo asociado y la aplicación del Derecho del trabajo. Valencia: Tirant lo Blanch y Universitat de Valencia, 28-29, 218-219.

Morínigo, S. (2011). Consultoría "Cobertura de Seguro Médico, de Vida y Jubilatorio para los Socios de las Cooperativas de Cooperativas de Trabajo Asociadas". Consorcio Intercooperativo. 10/10/2011. Informe final de Consultoría. Asunción:
Confederación Paraguaya de Cooperativas (CONPACOOP).

Organización Internacional del Trabajo (OIT) (2011). Seguridad social para la justicia social y una globalización equitativa. Discusión recurrente sobre la protección social (seguridad social) en virtud de la Declaración de la OIT 102 relativa a la justicia social para una globalización equitativa, 2011. En Conferencia Internacional del Trabajo, 100. reunión. Informe VI. Ginebra: OIT.

Organización Internacional del Trabajo (OIT) (2013). Informe IV. Empleo y protección social en el nuevo contexto demográfico. Conferencia Internacional del Trabajo, 102ª reunión. Ginebra: OIT.

Rodríguez Silvero, R. y Pei, P. (agosto de 2003). Seguridad Social. Descripción del Sistema Vigente de Jubilaciones y Pensiones en Paraguay. En Secretaría Técnica de Planificación, Informe final. Proyecto Planificación del Desarrollo Sostenible. Asunción: STP-PLANDES.

Ruesga Benito, S.M. (2009). El sistema social español en un marco de flexiseguridad. Análisis comparado de algunas experiencias europeas y lecciones para el caso español. España: Gobierno de España, Ministerio de Trabajo e Inmigración. Secretaría de Estado de Seguridad Social. Recuperado de: http://www.seg-social. es/prdi00/groups/public/documents/binario/143933.pdf (consultado el 17 de mayo de 2015).

Sánchez Martínez, M.T. (abril de 2007). El mutualismo de previsión social en España. En Revista de Economía Publica, Social y Cooperativa, (57). Centro Internacional de Investigación e Información sobre la Economía Pública, Social y Cooperativa (CIRIEC). Valencia: CIRIEC. 